\title{
The Prodigy Mind of a Genius and the Creative Process
}

\section{Nydia J Gutierrez*}

Studies in Neuroscience and Cognitive Psychology, Joint Degree with Massachusetts Institute of Technology Art, Culture, and Technology, Post Doctoral Degree Neuroscience, Artificial Intelligence, and Computational Modeling, Joint Degree with Massachusetts Institute of Technology Art, Culture, and Technology, USA

*Corresponding Author: Nydia J Gutierrez, Studies in Neuroscience and Cognitive Psychology, Joint Degree with Massachusetts Institute of Technology Art, Culture, and Technology, Post Doctoral Degree Neuroscience, Artificial Intelligence, and Computational Modeling, Joint Degree with Massachusetts Institute of Technology Art, Culture, and Technology, USA.
Received: May 02, 2019

Published: January 29, 2020

(C) All rights are reserved by Nydia J

\section{Gutierrez.}

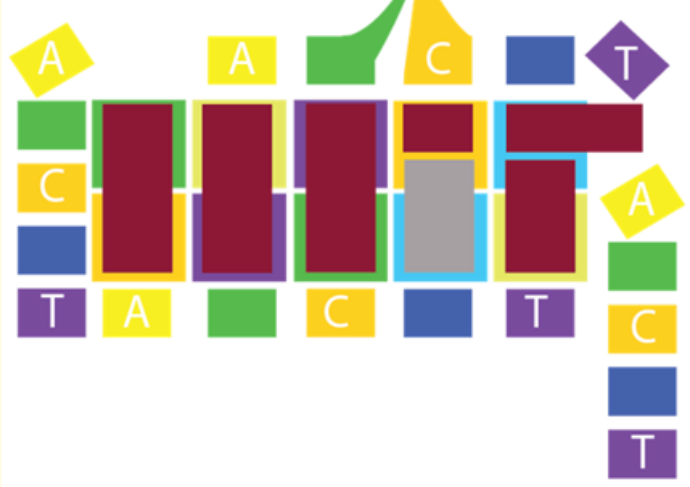

Figure 1

The prodigy mind of a genius

Color Memory, Neuroscience, and the Composition of Artificial Intelligence So what is the composition of a color memory? The ability to understand the composition and information of the visual feature of the human brain. Color memory is the capacity to view things based on a photographic mind mapping perception. The abstraction to understand the relation of neuroscience and the visual arts. The Newton methodology of the color theory and the Einstein capacity to think in numbers and space or time. So perhaps, it is important to understand the influence of human memory and the visual arts. The visual influence and formation of the various stages of the visual cortex. Neuroscience relates and embed into the visual arts. And artificial intelligence creates a powerful influence on the visual cognition of human visual memory. Therefore, there is the possibility to think in numbers and to recreate that visual capacity into space and time. So what is a prodigy mind? Is it the capacity to conjugate neuroscience and the visual arts, or to think in pictures [1].

We all ask, what makes a person gifted with a privilege and prodigy mind. The answer to this simplicity question starts with the study of neuroscience, spirituality, and the visual arts. The prodigy mind is the capacity to think in pictures, in the visual mental abstraction to neuroscience. A photographic memory results as a gift from the Holy Spirit. So the sequence of colors starts with the visual abstraction to spirituality. The perception to understand the visual image into a sequential of neurons. How it all translates into the design of luminance and patterns. The visual patterns that make possible mind mapping and the spectrum to a visual sequence wavelength [2].

The prodigy mind

In the Abstraction to Mind Mapping Visual Creative Neuroscience So what is giftedness and brilliance? The prodigy of a mind is design in the abstraction of a visual algorithm. The sequence to color defines how memory understands and interprets the visual cognitive hierarchy. Sensorial perception and abstraction is a true understanding to what and how we can think. So what does mind 
mapping engages and recreates into the visual algorithm to thinking. Creativity is a cognitive process that enhances the visual areas in the cortex to abstraction. The numerical and visual sequence to an order mind and how it recreates into the neuroscience cognitive process. Mathematics is another sense of spatial abstraction thinking that occurs in a prodigy mind or as a sense of a genius. Profound thinking determines that scholarly process that allows computational thinking in a nuclear systematic way [1].

The Prodigy Mind of a Genius: In the Abstraction to Mind Mapping Visual Creative Neuroscience The Prodigy Mind of a Genius mind is the Abstraction to Mind Mapping Visual Creative Neuroscience. Color memory and artificial intelligence do come together. It is the computational algorithm that defines another level of knowledge and intellectual learning. Computational modeling does create a visual abstraction to the recognition of knowledge and learning. The Prodigy Mind of a Genius is perhaps the visual colors of memories and neuroscience. The intellectual capacity to think with mathematical models and algorithm that define human intelligence. Creativity is another aspect that relates and correlates to human learning. Artificial Intelligence is another level of learning and understanding the brilliance of the human mind [2].

The prodigy mind of a genius

Computational Modeling and Artificial Intelligence The Prodigy Mind of a Genius from Computational Modeling and Artificial Intelligence abstraction. What is a Prodigy Mind and what makes a Genius? The simplicity of this question starts with the amount of learning. Natural Intelligence comes in many forms and diversification. The Prodigy of the mind and the capacity to associate different aspects of human cognition. This comes from Einstein to Van Goth or some other privilege mind that surpasses the standard of knowledge and learning. The brain enriches through education and the amount of learning you are to surpassed and accomplished. A Prodigy Mind has the capacity to cross disciples in such a way that becomes second nature. From pure mathematics to scientific and the genius's idea to generate new creativity. A One or Two Degree doesn't do it anymore. It is the ability to trespass conventional learning and generate new ideas [3].

Artificial Intelligence, a Brilliant Mind, and a whole new level of learning. Artificial Intelligence, a Brilliant Mind, and a whole new level of learning starts with the computational mind developing of visual neuroscience. Brilliance is the capacity to think in visu- al pictures that create and encompassed a new level of thinking. Creativity enhances the visual areas of the brain and this creates a cognitive visual area that recreates human intelligence. Artificial Intelligence has the capacity to connect and combined neuroscience with levels of creativity and robotics. This recreates human intelligence and a whole new level of learning and how that creates a visual human cognitive map. It is the recreation of a beautiful brilliant mind through visual learning and creative thinking. Neuroscience provides profound learning capacities that allows us to create a provide human learning. Meaningful test that help find a cure for Alzheimer mental disease [2].

Artificial Intelligence, a Brilliant Mind, and a whole new level of learning: The Creative Visual Process.

Artificial Intelligence, a Brilliant Mind, and a whole new level of learning encompass different areas of the visual cognitive process. Brilliance starts with a whole new level of thinking and with the spatial abstraction navigation. It is the start to the creative process and how it recreates the visual cognitive hierarchy. Neuroscience provides profound areas of research that enable the mind to cross disciples in the most outstanding manner to thinking and visual thinking. It is the recreation to a whole new level of human intelligence. Navigation starts with the visual cognitive computational hierarchy to what neuroscience is. Therefore, the essential creative method starts with cognitive thinking and the visual process [3].

\section{Conclusion}

So what is Brilliance? Brilliance is the representation of mathematical and creative visualization. It is the process to understanding visual perception and visual abstraction. A method that allows us to think in color memory and pictures. The sequence to discovery and curiosity. The design to mind mapping and the presentation of neuroscience, visual memory, and artificial intelligence. The incorporation of a higher knowledge that becomes lucid and represented in a visual impact. Brilliance also comes in a genetic investigation that leads to an experiment of visual language and mathematical patterns.

\section{Bibliography}

1. Garret B. Brain and Behavior: The cells that make us who we are. England, London; Sage Publications (2011).

2. Parker Steve. The Human Body. How the Brain and Vision Works. London, England; DK Publications (2015). 
3. McEntarffer R. AP Psychology: Brain Function and Structure, Hauppauge, New York; Barron's Educational Theory (2014).

\section{Assets from publication with us}

- Prompt Acknowledgement after receiving the article

- Thorough Double blinded peer review

- Rapid Publication

- Issue of Publication Certificate

- High visibility of your Published work

Website: https://www.actascientific.com/

Submit Article: https://www.actascientific.com/submission.php

Email us: editor@actascientific.com

Contact us: +919182824667 